\title{
PREVALENCIA DE MALNUTRICIÓN POR EXCESO ENTRE LOS ADOLESCENTES DE LOS LICEOS PRIVADOS DE MONTEVIDEO
}

\author{
PREVALENCE OF OVERWEIGHT AMONG ADOLESCENTS \\ OF PRIVATE HIGH SCHOOLS OF MONTEVIDEO \\ PREVALÊNCIA DE MÁ NUTRICCÃO POR EXCESSO ENTRE OS ADOLESCENTES
DE COLÉGIOS PARTICULARES DE MONTEVIDÉU
}

\author{
Isabel, Pereyra \\ Universidad Católica del Uruguay. Montevideo, Uruguay. \\ ipereyra@ucu.edu.uy \\ Carolina de León \\ Universidad Católica del Uruguay. Montevideo, Uruguay.
}

Recibido: 15/04/2016

\section{RESUMEN}

La Organización Mundial de la Salud ha identificado el sobrepeso y la obesidad como el quinto factor de riesgo para la salud en una escala global. El objetivo de este estudio fue evaluar la prevalencia actual de sobrepeso y obesidad entre los adolescentes escolarizados de 10 a 40 año de liceos privados de la ciudad de Montevideo. En cuanto a la metodología, se realizó la valoración del estado nutricional mediante la toma de peso y talla, con instrumentos acordes y calibrados. Los resultados arrojaron que del total de adolescentes $63 \%$ se clasifican como normopeso, $37 \%$ de los adolescentes presentó malnutrición por exceso y $0,4 \%$ emaciación. La obesidad fue mayor en los varones $13,7 \%$ contra $4,7 \%$ de las mujeres, diferencia estadísticamente significativa $(p=0,001)$. También existió asociación estadísticamente significativa entre el grado que cursa y el estado nutricional $(p=0,003)$. La población de adolescentes de primer grado presentó una prevalencia de obesidad $(16,7 \%)$ que duplica a la de cuarto $(8,5 \%)$ y a su vez es 3 veces mayor que la de segundo $(5,2 \%)$ y tercer grado $(5,0 \%)$. El presente estudio demostró la elevada prevalencia de sobrepeso y obesidad entre adolescentes escolarizados en los liceos privados de Montevideo. Cuando lo esperable para una población bien nutrida es que el $82 \%$ presente estado nutricional normal (IMC) Edad entre $-2 \mathrm{DE} a+1 \mathrm{DE}$ ) en el presente estudio solamente $63 \%$ estaban en esta situación.

Palabras clave: Adolescentes, Nutrición, Obesidad, Sobrepeso, Educación Primaria y Secundaria.

\section{ABSTRACT}

The World Health Organization has identified overweight and obesity as the fifth risk factor for health on a global scale. The aim of this study was to evaluate the prevalence of overweight and obesity among adolescents in high school from grade 1 to grade 4 of some private schools in Montevideo. The research assessed the nutritional status by measuring weight and height with calibrated instruments. It was found that $63 \%$ of the ado-

\author{
Aceptado: 27/05/2016
}

lescents are classified as having normal weight, $37 \%$ presented malnutrition by excess and $0.4 \%$ were severely underweight. Obesity was higher in males, $13.7 \%$ against $4.7 \%$ of the women, a statistically significant difference $(p=0.001)$. There was also a statistically significant association between the degree course and the nutritional status $(p=0,003)$. The population of teenagers of grade 1 presented a prevalence of obesity (16.7\%) that duplicates that of grade $4(8.5 \%)$ and is three times larger than that of grade $2(5.2 \%)$ and grade $3(5.0 \%)$. We found a high prevalence of overweight and obesity among adolescents in private high schools of Montevideo. When the expectation for a well-nourished population is that $82 \%$ will present a normal nutritional status (BMI/age between -2 to +1 ) in the present study only $63 \%$ were in this situation.

Keywords: Adolescents, Nutrition, Obesity, Overweight, Education, Primary and Secondary.

\section{RESUMO}

A Organização Mundial da Saúde identificou o sobrepeso e a obesidade como o quinto fator de risco para a saúde, em uma escala global. $\mathrm{O}$ objetivo deste estudo foi avaliar a prevalência atual do sobrepeso e a obesidade entre os adolescentes escolarizados do 1 음 4 을 Ano do ensino médio de colégios particulares da cidade de Montevidéu. Com relação à metodologia, foi realizada a avaliação do estado nutricional mediante o peso e o tamanho, com instrumentos adequados e calibrados. Os resultados determinaram que $63 \%$ do total de adolescentes são classificados como normopeso, $37 \%$ dos adolescentes apresentou má nutrição por excesso, e $0,4 \%$, emaciação. A obesidade foi maior no sexo masculino: $13,7 \%$ contra $4,7 \%$ no sexo feminino, diferença estatisticamente significativa $(p=0,001)$. Além disso, houve associação estatisticamente significativa entre o ano que cursa e o estado nutricional $(p=0,003)$, a população adolescente do 10 Ano apresentou uma prevalência de obesidade $(16,7 \%)$ que duplica à do $40(8,5 \%)$ e, por outro lado, é três vezes maior que a do 2 은 $(5,2 \%)$ e 3 o Ano $(5,0 \%)$. O presente estudo demonstrou a elevada prevalência do sobrepeso e da 
obesidade entre adolescentes escolarizados de colégios particulares de Montevidéu; enquanto era esperado, em uma população bem nutrida, que $82 \%$ apresentasse estado nutricional normal (IMC/idade entre $-2 \mathrm{DE} a+1 \mathrm{DE})$, no presente estudo, somente $63 \%$ estava nessa situação.

Palavras-chave: Adolescentes, Nutrição, Obesidade, Sobrepeso, Ensino Fundamental a Médio.

\section{INTRODUCCIÓN}

El estudio del estado nutricional y crecimiento longitudinal de un adolescente o grupo es un marcador dinámico de su estado físico y de salud. La adolescencia es una etapa clave en el desarrollo humano, es el periodo en el que se establecen los hábitos alimenticios, constituyendo la base del comportamiento alimentario para el resto de la vida. Los problemas alimenticios comunes en los adolescentes pueden conducir a trastornos tales como la bulimia, anorexia nerviosa, desnutrición, sobrepeso y obesidad; estos trastornos pueden ser causados por el sedentarismo, cambios en el estilo de vida y el autoconcepto que se refleja en la percepción errónea del cuerpo al querer parecerse a los arquetipos difundidos por los medios de comunicación.

El sobrepeso y la obesidad en todas las edades siguen aumentando en el comienzo de este nuevo milenio, no sólo en los países desarrollados sino también en los emergentes (1). La Organización Mundial de la Salud (OMS) ha identificado el sobrepeso y la obesidad como el quinto factor de riesgo para la salud en una escala global (2). En los países altamente desarrollados el sobrepeso es el tercer factor de riesgo más importante para la salud, detrás del consumo de tabaco y la hipertensión (1). Unos años atrás el sobrepeso era el décimo factor de riesgo a nivel mundial y el quinto en los países altamente desarrollados, de ahí que la OMS ha llamado a la problemática de malnutrición por exceso actual como "Epidemia mundial" (3). La consecuencia de la pandemia de obesidad es la expansión mundial de las grandes enfermedades crónicas del siglo XXI: enfermedad vascular, diabetes II, cáncer y aumento de mortalidad global (4).

La prevalencia de obesidad varía entre diferentes etnias y países (5). La publicación del NHANES 1999-2000 devela que el ritmo de progresión de la obesidad se ha acelerado: $64 \%$ de los adultos en EEUU padecen sobrepeso $u$ obesidad y $31 \%$ presentan un índice de masa corporal (IMC) $>30,0$ (6). Latinoamérica tampoco está ajena a la transición demográfica y nutricional. Las diferentes encuestas estiman que la prevalencia de sobrepeso es de $50 \%$ en las grandes urbes y la obesidad de $15 \%$ (7). Uruguay no escapa a la pandemia en la Primera y Segunda Encuesta Nacional de Sobrepeso y Obesidad (ENSO 1 y 2), se han detectado cifras muy altas: $54 \%$ de sobrepeso y obesidad (8). Los datos de la Primera Encuesta Nacional de Factores de Riesgo llevada a cabo por el Ministerio de Salud Pública, que toman como intervalo etario muestral 25-65 años, señalan también cifras epidémicas, $57 \%$ de sobrepeso y obesidad (9). Pero son más alarmantes los datos recabados en la 2 ㅇ Encuesta Nacional de Factores de Riesgo del año 2013 que muestran un aumento de 8 puntos porcentuales, lo que representa que el $64,7 \%$ de la población adulta en Uruguay presenta sobrepeso y obesidad (10).

A la vez, Uruguay presenta elevada morbilidad como consecuencia de las enfermedades crónicas relacionadas con la nutrición. En 2013 el 38,7\% presentó presión arterial elevada, mayor prevalencia respecto a $2006(9,10)$. La prevalencia de colesterol elevado disminuyó, de $29,2 \%$ a $23,1 \%$ (no estadísticamente significativo) $(9,10)$. La diabetes o glucemia elevada aumentó de 5,3\% en 2006 a 7,8\%, en $2013(9,10)$. La inactividad física en 2006 fue de 35,1\% y disminuyó significativamente en 2013 a 30,1\% $(9,10)$. La prevalencia del consumo diario de verduras y futas menor a la recomendación ( 5 porciones) aumentó de $84,9 \%$ a $90,8 \%$ en $2013(9,10)$. La obesidad es un grave problema de salud pública de la juventud de América, con tasas que se vienen más que triplicando en los últimos 30 años entre los adolescentes de edades comprendidas entre 12 y 17 años. Para el caso de Uruguay, la Segunda Encuesta Nacional de Salud Adolescente, realizada en el año 2012, dio como resultado que el $27 \%$ de los adolescentes de entre 13 y 15 años presentaba sobrepeso y el $7 \%$ obesidad (11).

Los adolescentes obesos tienen un mayor riesgo en la edad adulta de padecer obesidad y los problemas de salud asociados, como el cardiovascular, hipertensión, resistencia a la insulina, diabetes, hígado graso, disfunción ortopédica y psicosocial (12). Por estas razones, el principal objetivo del presente trabajo consiste en evaluar la prevalencia actual de sobrepeso y obesidad entre los adolescentes escolarizados de 1으 a 4음 año de liceos privados de la ciudad de Montevideo.

\section{METODOLOGÍA}

El presente estudio es observacional de tipo transversal. Para su realización se utilizó una base de datos primaria. El marco muestral fue la población de adolescentes que asisten a liceos privados de la ciudad de Montevideo. Es pertinente aclarar que aproximadamente la mitad de la población del Uruguay vive en Montevideo, ciudad capital y el resto en los 18 departamentos del país. Se encuestó a 671 estudiantes, siendo esta muestra representativa. Se realizó un muestreo aleatorio estratificado con asignación proporcional por conglomerados. La selección de los liceos se realizó con probabilidad proporcional a la cantidad de estudiantes que asisten al mismo. Luego, dentro del centro educativo se seleccionó un grupo de cada grado escolar, aplicándose un censo al interior de cada uno. Se trabajó sobre la base de información censal de la matrícula inicial del año 2011, según liceos de Montevideo, del Departamento de Estadística de Educación Secundaria. Las entrevistas fueron en los centros educativos, previa firma del consentimiento informado por los padres o responsables de los adolescentes. Se preservó el anonimato de los sujetos investigados, asignándoles un código automáticamente. Se realizó la valoración del estado nutricional mediante la toma de peso y talla, con instrumentos 
acordes y calibrados. El registro de peso y talla se hizo sin calzado ni abrigo. La antropometría estuvo a cargo de Licenciados en Nutrición entrenados en las características del estudio. El trabajo de campo se llevó a cabo entre octubre de 2012 y mayo de 2013. La investigación se realizó bajo la responsabilidad de la Asociación Uruguaya de Dietistas y Nutricionistas (AUDYN). El financiamiento del proyecto estuvo a cargo de la empresa Bimbo- Uruguay.

En cuanto al análisis estadístico, el ingreso digital de datos se realizó a través de EPIDATA 3.0. Para el análisis estadístico se utilizó el software SPSS v. 22.0. Los datos antropométricos se procesaron con el software WHO AnthroPlus. Las mediciones antropométricas fueron evaluadas a través los indicadores Índice de Masa Corporal para la Edad (IMC/Edad) y Talla/Edad (T/ Edad) y se compararon con el patrón de referencia de la OMS (13). Se clasificó Emaciado (IMC/Edad <-2DE), Normopeso (IMC/Edad >=-2DE y <=+1 DE). Para malnutrición por exceso se consideró sobrepeso (IMC/Edad>+1DE) y obesidad (IMC/ Edad $>+2 D E$ ). Los resultados se expresaron en proporciones de frecuencias y estadísticos de resumen y de dispersión. Para testear la existencia de diferencias significativas se utilizaron tests de comparación de medias y chi cuadrado. Se consideró significancia estadística cuando el valor $p<0,05$.

\section{RESULTADOS}

En cuanto a la descripción de la población estudiada, el total de adolescentes a los cuales se les realizó la valoración del Estado Nutricional ( $\mathrm{n}: 671$ ), $47 \%$ fueron mujeres y $53 \%$ varones. La mediana de edad fue 14 años y el rango intercuartílico (RIC) de 1 año. Según grado escolar, el $28,7 \%$ perteneció a primer año de secundaria, el $27,5 \%$ a segundo año, el $23,6 \%$ a tercer año y el $20,2 \%$ a cuarto año o primer año de bachillerato. Para el estado nutricional de los adolescentes se estudiaron los valores de peso, talla e IMC/Edad comparando los valores por sexo. No se observan diferencias estadísticamente significativas entre varones y mujeres para IMC $(p=0,478)$, pero sí para talla y para peso.

Tabla 1. Edad, peso, talla e IMC de los estudiantes de secundaria investigados

\begin{tabular}{|c|c|c|c|c|c|c|c|}
\hline & Varones & & Mujer & & Total & & Valor $p^{*}$ \\
\hline & Media & $\mathrm{DE}$ & Media & $\mathrm{DE}$ & Media & $\mathrm{DE}$ & \\
\hline $\begin{array}{l}\text { Peso } \\
\text { (kg) }\end{array}$ & 59,00 & 13,15 & 54,73 & 10,00 & 56,67 & 11,29 & 0,001 \\
\hline $\begin{array}{l}\text { Talla } \\
(\mathrm{m})\end{array}$ & 1,64 & 0,10 & 1,59 & 0,07 & 1,62 & 0,09 & 0,001 \\
\hline IMC & 21,75 & 3,76 & 21,56 & 3,25 & 21,59 & 3,32 & 0,478 \\
\hline
\end{tabular}

*Comparación de medias

Los varones son en promedio $5 \mathrm{~cm}$ más altos $(p=0,001)$ y 2,3 kg más pesados que las mujeres $(p=0,001)$. Según el indicador IMC/Edad, se puede observar que el $63 \%$ de los adolescentes se clasifican como normopeso, $37 \%$ presentó malnutrición por exceso y $0,4 \%$ emaciación.
Tabla 2. Clasificación del estado nutricional de los adolescentes estudiados según IMC/Edad

\begin{tabular}{lcccccc} 
& \multicolumn{2}{c}{ Varones } & \multicolumn{2}{c}{ Mujeres } & \multicolumn{2}{c}{ Total } \\
\hline & FA & FR\% & FA & FR\% & FA & FR\% \\
Emaciado & 3 & 0,6 & 0 & 0,0 & 3 & 0,4 \\
Normal & 204 & 58,3 & 216 & 67,9 & 420 & 62,6 \\
Sobrepeso & 97 & 27,4 & 87 & 27,4 & 184 & 27,5 \\
Obesidad & 49 & $13,7^{*}$ & 15 & $4,7^{*}$ & 64 & 9,5 \\
Total & 353 & 100,0 & 318 & 100,0 & 671 & 100,0 \\
\hline
\end{tabular}

$*_{p}=0,001$

De esta muestra representativa, quienes presentan malnutrición por exceso son 248 de un total de 671 . Al analizar como se comporta la población estudiada respecto a la referencia OMS para el indicador IMC/Edad, se observa claramente una alta prevalencia de adolescentes con malnutrición por exceso (Gráfico 1).

Al estudiar las diferencias por sexo, el grupo de mujeres con estado nutricional normal supera en un $10 \%$ esta condición en los varones. La obesidad fue mayor en los varones en $13,7 \%$ contra un $4,7 \%$ de las mujeres, diferencia estadísticamente significativa ( $p=0,001$ ) (ver Tabla 2). Este asspecto que se puede visualizar cuando se compara la población estudiada, diferenciada por sexo, con la referencia (Gráfico 2).

Un $40 \%$ de los adolescentes de menor edad (11 a 14 años) estuvieron distribuidos por encima de $1 \mathrm{DE}$, en cuanto a IMC/ Edad. En cambio, de los mayores (15 a 17 años), 27\% estaban en esa situación. La media del puntaje $z$ de IMC/Edad fue de 0,74 para el grupo de 11 a 14 años y de 0,45 para el grupo de 15 a 17 años, siendo esta diferencia significativa $(p<0,001)$. También existió asociación estadísticamente significativa entre el grado que cursa y el estado nutricional $(p=0,003)$. La población de adolescentes de primer grado presentó una prevalencia de obesidad $(16,7 \%)$ que duplica a la de cuarto $(8,5 \%)$ y a su vez es 3 veces mayor que la de segundo $(5,2 \%)$ y tercer grado $(5,0 \%)$.

Tabla 3. Distribución porcentual de los adolescentes según grado escolar y prevalencia de obesidad

\begin{tabular}{lccc}
\hline Grado escolar & \multicolumn{2}{c}{ Estado Nutricional } & Total \\
& Sin Obesidad & Con Obesidad & \\
1 ㅇ & $83,3 \%$ & $16,7 \%$ & $100 \%$ \\
2 o & $94,8 \%$ & $5,2 \%$ & $100 \%$ \\
3 ㅇ & $95,0 \%$ & $5,0 \%$ & $100 \%$ \\
4 ㅇ & $91,5 \%$ & $8,5 \%$ & $100 \%$ \\
Todos & $90,5 \%$ & $9,5 \%$ & $100 \%$ \\
\hline
\end{tabular}

La emaciación observada en el $0,4 \%$ de los estudiantes es inferior al porcentaje esperado $(2,3 \%)$ en una población bien nutrida, no existiendo diferencias estadísticamente significativas entre sexos, ni edad.Respecto a la estatura, prácticamente todos los individuos (99\%) presentaron una talla adecuada para 
su edad y sexo, a partir de los criterios de la referencia de la OMS (Gráfico 3).

\section{DISCUSIÓN}

El presente estudio demostró la elevada prevalencia de sobrepeso y obesidad entre adolescentes escolarizados en los liceos privados de Montevideo. Cuando lo esperable para una población bien nutrida es que el $82 \%$ presente normopeso, en el presente estudio solamente $63 \%$ estaban en esta situación. Esto significa que prácticamente $40 \%$ de los adolescentes escolarizados en los liceos privados de Montevideo presentan algún grado de exceso de peso.

Teniendo en cuenta lo anterior la situación es preocupante, porque se conoce que el $50 \%$ de los adolescentes que presentan obesidad serán obesos en la vida adulta y a su vez el sobrepeso y obesidad constituyen uno de los principales factores de riesgo para muchas de las Enfermedades no Trasmisibles (ENT) (12). La constitución de la obesidad en edades tempranas como una verdadera pandemia mundial está recién comenzando a mostrar sus reales consecuencias a nivel de la salud integral. Aparecen cada vez más precozmente ENT y ya se observan prevalencias preocupantes desde la infancia temprana (14-15). En Uruguay, hay pocos datos representativos sobre la prevalencia de la obesidad en los adolescentes y los existentes no son todos comparables, porque se utilizaron diferentes definiciones de obesidad. La elevada prevalencia de exceso de peso encontrada en el presente estudio está acorde con los resultados de la II Encuesta Nacional de Salud Adolescente, de adolescentes escolarizados de 13 a 15 años de Liceos Públicos y Colegios Habilitados, del año 2012, en quienes el exceso de peso (IMC) Edad >+1DE) fue de $33,6 \%$ (11).

En el presente estudio los adolescentes varones presentaron mayor prevalencia de obesidad que las niñas. Estos resultados se diferencian de lo hallado en la II Encuesta Nacional de Salud Adolescente, donde no se encontraron que las diferencias fueran estadísticamente significativas en los porcentajes de estudiantes con sobrepeso y obesidad según sexo (11). Sin embargo, Moreno et al refiere que tanto en Europa como en Estados Unidos es sostenido el aumento del sobrepeso y la obesidad entre los adolescentes y se acompaña de una diferencia de género, siendo más prevalente en varones (16). El estudio AVENA también presenta claramente el problema de la prevalencia de la malnutrición por exceso en los adolescentes españoles. Al igual que en el presente estudio, son más afectados los varones que las niñas por esta problemática $(25,6 \%$ y $19,1 \%$ respectivamente) (17). Otro estudio realizado en Florianópolis, Brasil, entre adolescentes de 11 a 14 años de edad, mostró que la prevalencia de sobrepeso y obesidad fue de $19,3 \%$ en el total, también con mayor prevalencia en varones $(24,5 \%$ y $14,5 \%$ en niñas) (18). Según los rangos de edad, $40 \%$ de los adolescentes de menor edad presentaron sobrepeso y obesidad, en comparación con $27 \%$ en los adolescentes mayores. Lo mismo ocurre en la II Encuesta Nacional de Salud Adolescente, del año 2012, realizada a nivel nacional, donde los estudiantes mayores de 16 años muestran una prevalencia de sobrepeso menor respecto a los de 13 a 15 años (11). Esta situación puede explicarse porque la velocidad de crecimiento en talla que implica la pubertad pueda devenir en mejor relación del peso para la altura de los jóvenes. Por otra parte no se puede dejar de considerar que exista una tendencia al agravamiento de la situación de sobrepeso y obesidad desde edades más tempranas de la vida, sobre la que se hace imperioso actuar. Según Moreno et al, la tasa de cambio de la prevalencia de sobrepeso y obesidad ha

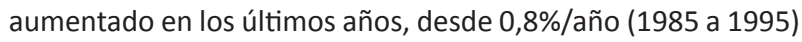
a 2,33\%/año (1995 a 2000-2002) en varones y desde 0,5\%/año (1985 a 1995) a 1,83\%/año (1995 a 2000-2002) en mujeres, lo cual apunta a la existencia de una verdadera "epidemia" de obesidad en los adolescentes (16). Está demostrada la relación de sobrepeso y obesidad con factores de riesgo para ENT. Diferentes estudios han confirmado que en las últimas décadas se ha producido un alarmante incremento de la prevalencia de estos factores de riesgo en la población adolescente. Los estudios longitudinales han encontrado relación entre obesidad durante la adolescencia y la presencia de enfermedad cardiovascular y diabetes en la vida adulta (16).

\section{CONSIDERACIONES FINALES}

La mayoría de los estudios sobre el estado nutricional entre los adolescentes concluyen que son necesarias intervenciones para mejorar la nutrición y deben ir acompañadas de la promoción del aumento de la actividad física. La adolescencia es considerada una segunda ventana de oportunidad para intervenir, no sólo en aspectos sociales y del desarrollo sino también nutricionales. Las herramientas para intervención en salud de los adolescentes deberían focalizarse en poblaciones específicas según variables socio demográficas y económicas para aumentar la eficacia de estas intervenciones. La adolescencia es una etapa única en la vida por lo tanto se considera que la educación sanitaria de los adolescentes debe permitir al individuo comprender lo que es positivo en su persona para que pueda desarrollar otros aspectos al máximo. No se deben forzar modelos de comportamiento sino ofrecer y fomentar una cultura basada en estilos de vida "a realizar" en vez de estilos de vida "a evitar" (19). También existe consenso en que el instituto educativo es el lugar idóneo para realizar intervenciones de educación nutricional en que estén involucrados no sólo los adolescentes, sino también las familias y los docentes (20).

En Uruguay ha habido avances en cuanto a determinar los lineamientos sobre la venta de alimentos y bebidas en los centros educativos con énfasis en el control de densidad calórica, grasas, azúcares y sal. Esta estrategia podría contribuir a las acciones de intervención en el ambiente al que se exponen los adolescentes cotidianamente (21). Los resultados del presente estudio deben ser considerados a la luz de ciertas limitaciones. Primero, reconocer que es una debilidad no haber podido valorar el estado de desarrollo de los adolescentes con el objetivo de incluir este dato relativo al grado de maduración en la evaluación nutricional. Segundo, la naturaleza de ser un estudio 
transversal no permite obtener relaciones de causalidad que pueden ser de gran utilidad a la hora de planificar acciones de prevención de malnutrición por exceso. A pesar de estas limitaciones, el presente estudio tiene fortalezas propias. La muestra, al ser representativa de los adolescentes de los liceos privados de la mayor concentración urbana del país y la amplia tasa de respuesta, permite generar resultados confiables. Además este estudio constituye un aporte al conocimiento del estado nutricional de los adolescentes del país debido a que hay escasos publicaciones sobre esta población con esta representatividad muestral.

\section{CONFLICTO DE INTERESES}

El proceso de realización del trabajo hasta el primer informe macro y de devolución a cada institución participante fue financiado por la Empresa Panificadora BIMBO del Uruguay SA a través de la Asociación Uruguaya de Dietistas y Nutricionistas. Las autoras declaran haber tenido independencia de la fuente de financiamiento y no tener conflictos de intereses relacionados con el contenido de esta publicación.

\section{REFERENCIAS BIBLIOGRÁFICAS}

1. Popkin BM, Gordon-Larsen P. The nutrition transition: worldwide obesity dynamics and their determinants. Int J Obes Relat Metab Disord 2004; 28 Suppl 3: S2-9.

2. WHO. Global health risks: mortality and burden of disease attributable to selected major risks. Genf: WHO; 2009

3. WHO. Obesity: preventing and managing the global epidemic. Report of a WHO Consultation. WHO Technical Report Series No. 894. Genf: WHO; 2000.

4. Calle EE, Thun MJ, Petrelli JM, Rodriguez C, Heath CW. Body mass index and mortality in a prospective cohort of US adults. N Engl J Med 1999; 341: 1097-105.

5. Martínez JA, Moreno B, Martínez-González MA. Prevalenceof obesity in Spain. Obes Rev 2004; 5: 171-2.

6. Flegal KM, Carroll MD, Ogden CL, Johnson CL. Prevalence and trends in obesity among US adults, 1999-2000. JAMA 2002; 288 (14): 1723-7.

7. Uauy R, Albala C, Kain J. Obesity trends in Latin America: transiting from under-to overweight. J Nutr 2001; 131: 893-9.

8. Pisabarro R y cols. Segunda Encuesta Nacional de Sobrepeso y Obesidad (ENSO 2) adultos (18-65 años o más). Rev Med Urug 2009; 25: 14-26

9. Ministerio de Salud Pública. Dirección General de la Salud. División Epidemiología. 1a Encuesta Nacional de Factores de Riesgo de Enfermedades Crónicas No Transmisibles. Montevideo, 2009; 29-30

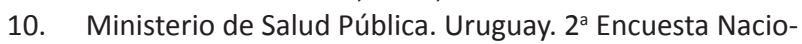
nal de Factores de Riesgo de Enfermedades Crónicas No Transmisibles. 2013. [Internet]. [citado Jul 2015]. Dis- ponible en: http://www.msp.gub.uy/noticia/segundaencuesta-nacional-de-factores-de-riesgo-de-enfermedades-cr\%C3\%B3nicas-no-transmisibles

11. MSP. MIDES. JND. OPS. Segunda Encuesta Nacional de Salud Adolescente. Uruguay. 2012. [Internet]. [citado Jul 2015]. Disponible en www.msp.gub.uy/publicacion/ adolescencias-un-mundo-de-preguntas-ii-encuestamundial-de-salud-adolescente-gshs-2012.

12. Baker JL, Olsen LW, Sorensen TI. Childhood body-mass index and the risk of coronary heart disease in adulthood. New Engl J Med. 2007; 357: 2329-37.

13. Organización Mundial de la Salud. Departamento de Nutrición para la Salud y el Desarrollo. Patrones de Crecimiento del Niño la OMS: Curso de Capacitación sobre la evaluación del crecimiento del niño. Washington: OMS, 2008

14. MSP, MIDES, RUANDI, UNICEF. Bove I, et al. Encuesta Nacional sobre estado nutricional, prácticas de alimentación y anemia en niños menores de dos años. 2011. [Internet]. [citado Ago 2015]. Disponible en: http:// www.unicef.org/uruguay/spanish/encuesta_lactancia_ uruguay2011_web.pdf.

15. Pastén A, Peñalillo T, Navarro J. Diagnóstico nutricional de población escolar en una isla de la VIII región. Rev Chil Pediatr 2014; 85 (2): 183-187

16. Moreno LA et al. Assessing, understanding and modifying nutritional status, eating habits and physical activity in European adolescents: The HELENA (Healthy Lifestyle in Europe by Nutrition in Adolescence) Study. Public Health Nutrition; 2007. 11 (3), 288-99

17. Wärnberg J, Ruiz JR, Ortega FB, Romeo J, González-Gross $M$, Moreno LA et al Estudio AVENA* (alimentación y valoración del estado nutricional en adolescentes). Resultados obtenidos 2003-2006. [Internet]. [citado Mar 2015]. Disponible en: http://www.estudioavena.es/downloads/ Publicaciones/WarnbergJ_2006Pediatrlnt.pdf.

18. Benedet J, de Assis MA, Calvo M, de Andrade D. Overweight in adolescents: exploring potential risk factors. Rev Paul Pediatr 2013; 31 (2): 172-81

19. The AVENA Group. Alimentación y valoración del estado nutricional de los adolescentes españoles. Evaluación de riesgos y propuestas de intervención. Nutr Hosp 2003; 18(1): 15-28

20. Macías Matos C, Días Sánchez ME et al. Estilos de Vida, sobrepeso y obesidad en adolescentes de enseñanza media de La Habana. Rev EspNutr Hum Diet. 2012;16(2):45:53.

21. Ministerio de Salud Pública. Programa Nacional de Nutrición. Lineamientos para la venta y publicidad de alimentos en centros de enseñanza primaria y secundaria públicos y privados del país. Documento Técnico. Montevideo. Marzo 2014. [Internet]. [citado Dic 2014]. Disponible en: http://www.msp.gub.uy/publicaci\%C3\%B3n/ lineamientos-para-la-venta-de-alimentos-y-bebidas-enlos-centros-educativos. 
Gráfico 1. Curva de IMC/Edad de la población estudiada comparada con la referencia de la OMS.

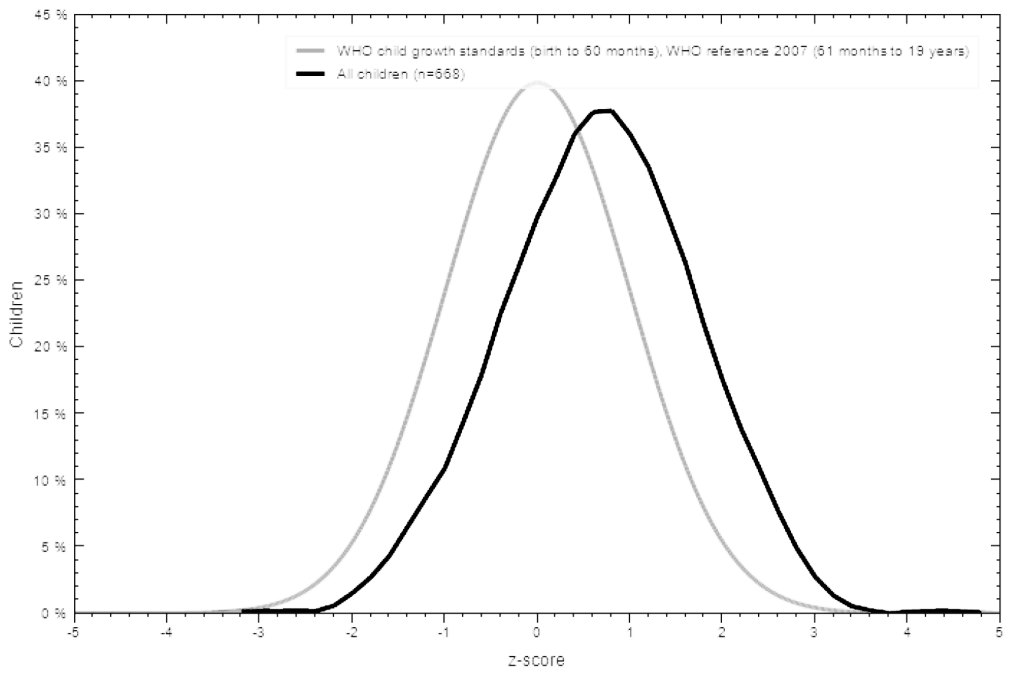

Gráfico 2. Curva de IMC/Edad de la población estudiada comparada con la referencia de la OMS, discriminada por sexo

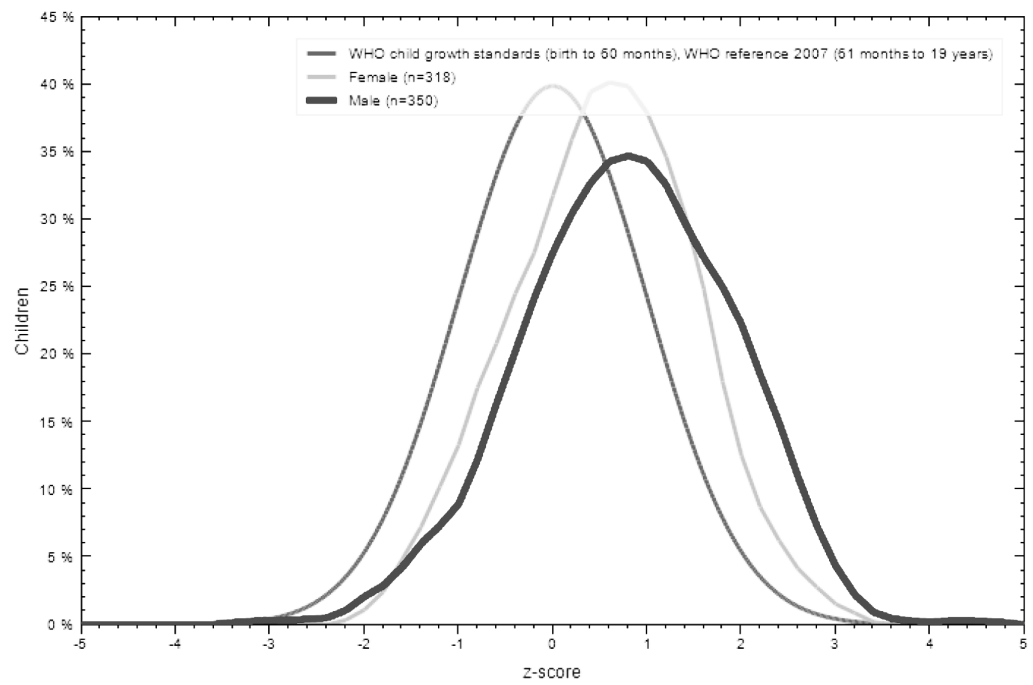

Gráfico 3. Talla/Edad de la población estudiada comparada con la referencia de la OMS

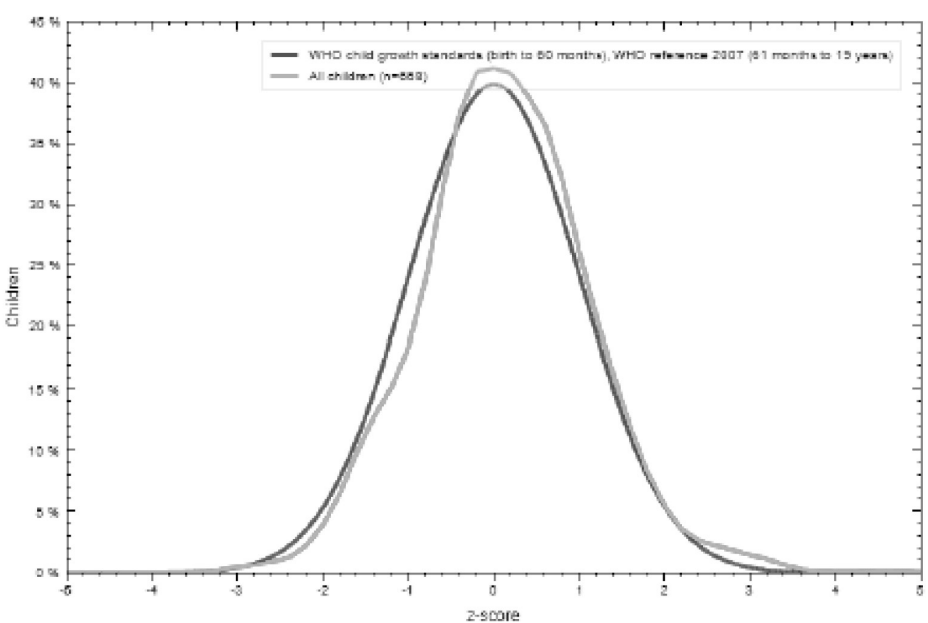

